\title{
Personalisation of the Multimedia Content Delivered to Mobile Device Users
}

\author{
Arghir-Nicolae Moldovan and Cristina Hava Muntean, Member, IEEE
}

\begin{abstract}
People using mobile devices for studying multimedia based educational content are often on the move and thus rely solely on their device battery power supply. When battery power runs low, they have to stop their activities, significantly reducing their learning outcomes and their satisfaction. This paper proposes a solution to perform the personalisation of the multimedia educational content, based both on the learner profile and on the available power resources on the device used. The solution aims to increase the battery life without affecting learner's quality of experience. Experimental results show that the battery life can be increased by changing streaming related parameters while preliminary subjective tests have assessed their impact on end user perceived quality of the multimedia clip.
\end{abstract}

Keywords-multimedia devices, adaptive multimedia clips, device battery

\section{INTRODUCTION}

$\mathrm{I}^{\mathrm{t}}$ is now considered reasonable to have high quality multimedia-based educational content delivered via wireless networks to mobile devices such as PDAs, smart phones, laptops. Already an increasing number of e-learning environments have improved their e-learning applications in order to support educational content display on various types of limited screen size devices.

Often learners using mobile devices, also called mlearners, are on the move and they rely only on the device battery power supply while they are engaged in a learning session. Since device display, CPU and wireless network card are major consumers of the device battery power when processing the resource intensive multimedia content, often situations occur when learners have to stop activities performed on the device (e.g. visualizing an educational multimedia clip) due to low power levels. This significantly affects their learning capabilities and learner Quality of Experience (QoE), negatively affecting learning outcome. In this context, there is a need for a novel power saving solution that would enable m-learners using devices with low battery power levels to visualize the entire multimedia-based educational content streamed during a learning session.

Manuscript received April 9, 2009. This work was supported by Science Foundation Ireland.

A.-N. Moldovan is with the School of Computing, National College of Ireland, Mayor Street, Dublin 1, Ireland (e-mail: amoldovan@student.ncirl.ie).

C. H. Muntean is with the School of Computing, National College of Ireland, Mayor Street, Dublin 1, Ireland (e-mail: cmuntean@ncirl.ie).
This paper proposes a solution to extend the functionality of traditional adaptive learning applications, considering the available power resources of the mobile device along with other factors in the content personalisation process. This solution, called Battery Aware Adaptive M-Learning System (BAAMLS), uses a power model based on mobile device characteristics such as battery properties and its state, network connectivity type, display characteristics, sound level, etc. The personalisation involves varying the parameters of the multimedia content that is being streamed, such as the encoding technique or the video resolution. This decreases power consumed by the mobile device to retrieve, decode and display the multimedia content. The personalisation process will be applied only if the playtime of the requested media content is higher than the remaining battery lifetime. As direct consequence a version of multimedia content that allows for streaming and playing of the whole clip on the device is delivered but at the same time is qualitative enough to not affect the learner QoE.

The paper is structured as follows. In section two, research efforts in the area of adaptive learning and notable power saving solutions are listed. Section three presents experimental testing and comparative results which show that various streaming parameters have an impact on the power consumption. The paper continues with the architecture of the proposed solution and finishes with conclusions and future work.

\section{RELATED WORK}

As wireless technologies are competing with wired technologies for content delivery and mobile devices became available to an increasing number of people, there is an inevitable trend that sees e-learning evolving towards mobile learning (m-learning). M-learning systems [1] provide support for access to educational content anytime, anywhere and from many devices. Various experiments have demonstrated that an adaptive approach can be effective when rich educational content is delivered to PDAs [2] or iPods [3]. However, how mobile devices can accurately detect the environment conditions (e.g. background noise, ambient light level, etc.) without excessive use of external sensors, how wearable devices can be used without user discomfort (e.g. out of battery power case) and how users' privacy and personal integrity is maintained when location-aware technology is used are research questions currently under investigation [4], 
[5].

Most of the research in the area of e-learning has concentrated on delivering personalised educational content tailored to individuals or groups based on learner characteristics (e.g. knowledge, preferences). More recently research focused on proposing solutions for multimedia content adaptation according to learner device type and characteristics [6], [7], network type and conditions [8] and user Quality of Experience (QoE) [9]. None of the research in the area has assessed how device battery affects the learner and learning capabilities mainly in a low power condition and did not consider the learner device battery level in the personalisation process of the multimedia content apart of other device characteristics.

Although the characteristics of device resources such as screen size, memory capacity, CPU processing power and network bandwidth have improved exponentially in recent years allowing for high quality personalized multimedia clips to be delivered over wireless connections and displayed on the device, batteries have fallen behind in terms of development. Combination of multimedia tasks that mainly use screen and speakers, CPU and memory used for decoding the multimedia stream and the streaming process of media clip that uses the Wireless Network Interface Card (WNIC) consumes the power in the battery very quickly. In particular, WNIC has been shown to be one of the most power hungry parts of the mobile device [10]. Therefore, mobile devices have not been designed to maintain such power intensive tasks for a long period of time, and if the battery depletes before a task is completed, learner satisfaction is significantly lowered.

Current research in the area of multimedia streaming investigates various power saving solutions [11]. The proposed solutions can be classified in the following categories:

- Power saving in the reception stage of the multimedia stream. These solutions look at sending and receiving of data and mainly focus on WNIC. They include techniques such as sending frames in a burst based on the network traffic shape instead of sending them individually and thus reducing data waiting time for the device [12] and client side prediction scheme for suspending communication and switching the WNIC to a temporary sleep state [13].

- Power saving in the decoding stage. The multimedia stream is received and decoded by the device. The encoding data technique will impact the amount of battery power required to decode the data.

- Power saving in the playing stage. Based on the media clip type, the screen, the speakers or a combination of the two will be used to play the multimedia. The majority of the power saving techniques in this area have focused on the device screen and involve optimizing backlight power consumption [14], extending the Dynamic Luminance Scaling (DLS) to cope with transflective LCD panels that can operate with or without backlight [15] depending on the battery level and ambient luminance. Since a built-in brightness control exists on mobile devices and in general the user can control the speakers and screen settings little research in this area has been performed.

Considering the existing research in the areas of (m-) learning and power saving in multimedia streaming, it is important to note that this research bridges the two areas by complementing a learner oriented educational solution that delivers multimedia content with a device battery power saving solution. This novel approach supports learning activities in low battery conditions allowing for the entire visualisation of the multimedia clip during a learning session.

\section{EXPERIMENTAL BATTERY CONSUMPTION TESTING}

Often, m-learning applications imply delivery of media rich educational content. Playing a video that is streamed over the wireless network to a mobile device is an energy intensive task. In this situation, a large quantity of the power drained from the battery is consumed by the WNIC for packets reception and processing (1), by the CPU and memory for decoding and generating the video frames (2), and by the display and speaker(s) for displaying the video (3). (1), (2), and (3) correspond in a good measure to the three stages of multimedia streaming: data reception, decoding and playing respectively.

Mobile device users have a high level of control on the device settings like screen brightness or sound level. Changing these settings in order to save power can have a negative impact on users QoE.

On the other hand, users have reduced control on how data is received and on the amount of resources necessary to decode and play the video.

Therefore, various experimental tests were conducted in order to investigate the effect of (1) and (2) on the battery power consumption and the results are summarised in this section.

\section{A. Experimental Setup}

A test bed similar to the one presented in Figure 1 was used. A Lenovo laptop with $2 \mathrm{GHz}$ Intel Core 2 Duo processor and 2 GB of RAM memory running Microsoft Windows Vista is used as a multimedia streaming server. The server is connected to an Access Point (AP), using an Ethernet link. The client machine consists of a Dell Axim PDA with 512 MHz Intel PAXA270 processor and an IEEE $802.11 \mathrm{~b}$ wireless network card, running Microsoft Windows Mobile 5.The client machine requests and plays in real-time the video

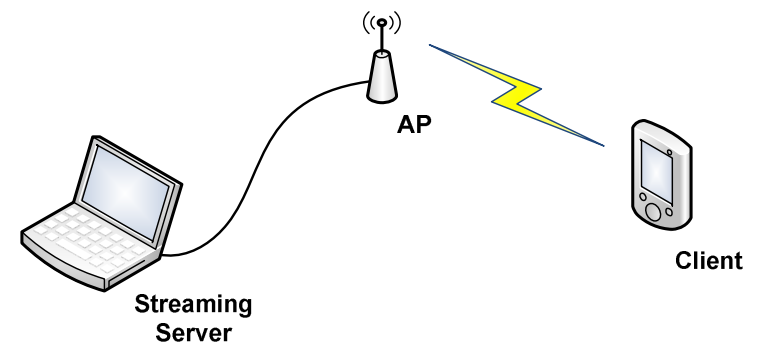

Fig. 1. Test setup architecture 
TABLE I

POWER CONSUMPTION OF DifFERENT VIDEO CODECS

\begin{tabular}{|c|c|c|c|}
\hline $\begin{array}{c}\text { Video Codec / } \\
\text { Container Type / } \\
\text { Streaming Protocol }\end{array}$ & $\begin{array}{c}\text { Playback Time } \\
50 \% \text { Battery Left } \\
\text { [min] }\end{array}$ & $\begin{array}{c}\text { Playback Time } \\
\text { 30\% Battery Left } \\
\text { [min] }\end{array}$ & $\begin{array}{c}\text { Avg. Current Drain } \\
50 \% \text { Battery Left } \\
{[\mathrm{mA}]}\end{array}$ \\
\hline \multicolumn{4}{|c|}{ When Streaming and playing a media clip over a wireless network } \\
\hline WMV 9 / ASF /MMS & 41.1 & 29.2 & 586 \\
\hline MPEG4 Part 2/ MPEG-TS / UDP & 43.4 & 30.7 & 535 \\
\hline H.263 / FLV / RTMP & 46.5 & 33.3 & 526 \\
\hline \multicolumn{4}{|c|}{ When playing the video clip stored locally on the device } \\
\hline H.264 / MP4 & 65.9 & 49.7 & 332 \\
\hline WMV 9 / WMV & 66.7 & 51.7 & 348 \\
\hline MPEG4 Part 2/ AVI & 81.4 & 59.6 & 286 \\
\hline H.263 / FLV & 85.5 & 65.7 & 289 \\
\hline
\end{tabular}

streamed over the wireless network.

In order to reduce at the minimum the bandwidth fluctuations, the PDA was the only wireless device connected to the AP. The PDA was also maintained in a fixed position where the wireless signal strength was high and constant, for the entire period of time when testing was conducted.

A video sequence of 10 minutes length and with a reduced degree of motion content, corresponding to educational videos was used. For all the test cases, the sound was encoded on two channels, with an average bit rate of $32 \mathrm{Kbps}$ and a sampling rate of $22.050 \mathrm{~Hz}$. If not otherwise specified, the video was encoded using MPEG-4 AVC (H.264) compression, with an image size of $320 \times 240$ pixels, a frame rate of $24 \mathrm{fps}$ and an average video bit rate of $384 \mathrm{Kbps}$. The encoding operations were performed using Media Coder application [16].

Depending on the streaming type, following streaming applications were used: Adobe Flash Media Server (FMS) [17], Darwin Streaming Server (DSS) [18] and VideoLAN VLC [19].

On the client side, battery related information such as battery level, battery voltage, current consumption, battery temperature, were saved in a trace files using BattLog utility [20]. PDA screen brightness and volume levels were set to $100 \%$.

\section{B. Experimental Scenarios and Results}

Multimedia clip parameters such as video encoding technique, video image size, frame rate and bit rate, were considered and their effect on battery consumption was studied. Various versions of the studied video clip that would have different values of the studied parameters were created. Each video version was streamed to the mobile device and played locally in loop until the battery was completely discharged. The initial stage of the battery was $50 \%$ charged for all testing scenarios.

A first set of tests was conducted in order to assess the energy efficiency of four video compression techniques that are predominantly used for multimedia streaming: MPEG-4 AVC (H.264), H.263, WMV 9 and MPEG-4 Part 2. The container type and the streaming protocol used in each case are presented along with the results in Table I. H.264 streaming was performed using DSS, flash streaming (H.263) using FMS, while the other two (WMV 9 and MPEG-4 Part 2) using VLC.

Results show that by changing the video compression of the streamed video from H.264 to H.263 when the battery level drops below $31 \%$, significant improvements in battery life of approximately $35 \%$ can be achieved, which translates in 8.6 minutes of extra playing time. Results also show that by changing the video compression after battery level drops below $51 \%$, improvements of approximately $28 \%$ are achieved, or 10.1 minutes of extra playing time. In the second case the user will see 13.2 more minutes of lower quality than in the first case for only 1.5 minutes of extra playing time.

A new testing scenario has investigated the effect of WNIC on battery consumption. The videos used in the previous testing scenario were stored and played locally on the PDA device. An increase in battery life between 77\%, for WMV 9, and $101 \%$, for H.264, was achieved (see Table I) for the case when playing started at $30 \%$ battery power. Figure 2 shows that significant battery life improvement is achieved at low battery levels. For example, if the H.263 media clip version

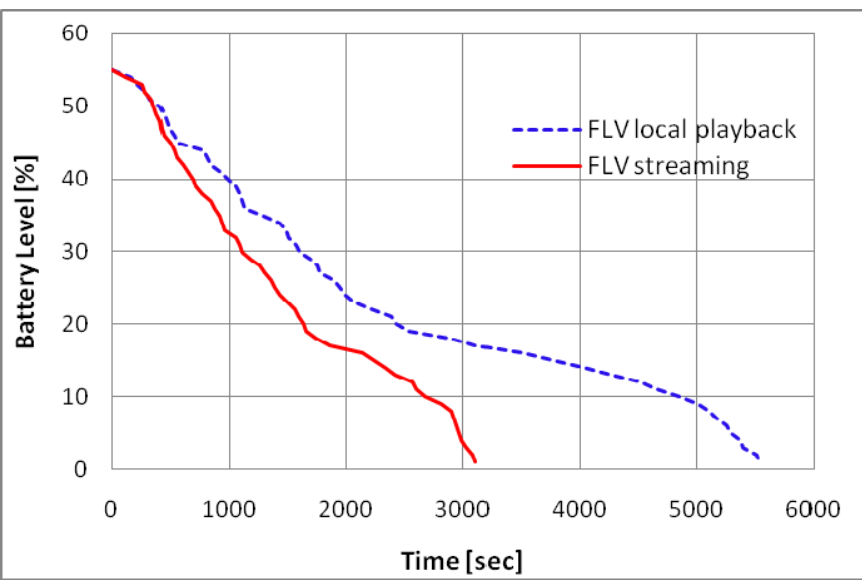

Fig. 2. H.263 (FLV) - Local Playback versus Streaming 
TABLE II

H.264 Streaming: The EFFect Of Quantitative EnCoding Parameters On BATtery Life

\begin{tabular}{|c|c|c|c|c|c|}
\hline $\begin{array}{l}\text { Video Resolution } \\
\text { [pixels] }\end{array}$ & $\begin{array}{c}\text { Frame Rate } \\
{[\mathrm{fps}]}\end{array}$ & $\begin{array}{c}\text { Avg. Video Bit Rate } \\
{[\mathrm{Kbps}]}\end{array}$ & $\begin{array}{c}\text { Playback Time } \\
50 \% \text { Battery Left } \\
\text { [min] }\end{array}$ & $\begin{array}{c}\text { Playback Time } \\
30 \% \text { Battery Left } \\
\text { [min] }\end{array}$ & $\begin{array}{c}\text { Avg. Current Drain } \\
50 \% \text { Battery Left } \\
{[\mathrm{mA}]} \\
\end{array}$ \\
\hline $480 \times 360$ & 24 & 384 & 33.6 & 23.2 & 715 \\
\hline $240 \times 180$ & 24 & 384 & 38.9 & 26.2 & 616 \\
\hline $320 \times 240$ & 24 & 384 & 36.4 & 24.7 & 669 \\
\hline $320 \times 240$ & 20 & 384 & 37.4 & 25.5 & 653 \\
\hline $320 \times 240$ & 24 & 512 & 35.8 & 24.3 & 677 \\
\hline $320 \times 240$ & 24 & 384 & 36.4 & 24.7 & 669 \\
\hline $320 \times 240$ & 24 & 192 & 38.4 & 26.0 & 653 \\
\hline
\end{tabular}

starts to play locally when the battery is only $50 \%$ charged, there will be an increase of approximately $84 \%$, or 39 minutes, in the playing time, compared to the situation when the video is streamed until the battery is completely discharged. By starting to play locally when there is $30 \%$ left of the battery, the improvement in playing time will be of $97 \%$ or 32.4 minutes.

Concluding this testing one can say that WNIC is responsible for up to $50 \%$ of the total battery consumption when receiving the streamed media clip.

A third set of tests was conducted in order to assess the effect of quantitative encoding parameters on battery consumption. For exemplification purpose the H.264 video compression format is presented in this paper. The video image size, the number of frames per second and the average video bit rate are the parameters considered in this testing scenario. Results (see Table II) show that these parameters have a lower impact on battery consumption. Improvements in battery life of 13\% (3 $\mathrm{min}), 15 \%$ (3.7 $\mathrm{min})$ and 7\% (1.7 $\mathrm{min})$ can be achieved by reducing the resolution from $480 \times 360$ pixels to $240 \times 180$ pixels, the frame rate from $24 \mathrm{fps}$ to $16 \mathrm{fps}$ and the video bit rate from $512 \mathrm{Kbps}$ to $192 \mathrm{Kbps}$ respectively. The above results were achieved by changing the media clip parameters when battery level dropped below $31 \%$.

\section{Preliminary Subjective Tests}

Subjective tests were conducted on a small number of participants in order to investigate how learners perceive the changes in video encoding parameters when video sequences are played locally on the mobile device. Preliminary results have shown that a decrease in video resolution, frame rate or bit rate, has a higher impact on the average user perceived quality than changing the video compression technique [21].

\section{BATTERY AWARE ADAPTIVE M-LEARNING SYSTEM} (BAAMLS)

This section proposes a solution - Battery Aware Adaptive M-Learning System (BAAMLS) - to extend the classic architecture of an Adaptive (E)-Learning System (ALS) in order to consider battery power level in the personalisation process of the educational content. The solution aims to assist the learners in low power situations, and increase the time learners can dedicate to their current learning activities, without affecting their QoE. This is done by maintaining a good balance between the quality of the multimedia content delivered and the amount of power saved.

\section{A. System Architecture}

The system consists of client-server architecture, presented at the block level in Figure 3. The main components on the server side include four models which store and update the information needed by the learning system, a Personalisation Engine (PE) and a Streaming Module (SM). The four models are: User Model (UM), Domain Model (DM), Adaptation Model (AM) and Power Model (PM). Apart of the PM, all the other components can be found in the classic architecture of an ALS.

The UM maintains relevant information about the users, gathered explicit (via registration) or implicit (through navigation), and is updated continuously. The information can be: demographic information (e.g. name, age, gender ...), knowledge level on the studied material, evaluation results, interests, content related preferences, etc.

The DM stores the educational content and is organised in a hierarchical structure of concepts, among which logical relationships exist. At the lowest level, each atomic concept corresponds to a specific piece of educational information which can be a text, an image or a media clip. In case of multimedia clips, the model was extended to allow different versions of the same clip to be associated with an atomic concept. Each version has at least one parameter, such as resolution, frame rate, bit rate or encoding technique, different of the others.

The AM consists of a set of condition-action rules (UP Rules) used to express the personalisation of the educational material based on the user profile. This model was extended with a new set of rules (BM Rules) that express the adaptation of the multimedia content based on a particular device battery model, in order to increase the battery life in low power situations.

Battery Models (BM) are stored and updated by the Power Model (PM), each one corresponding to a particular mobile 


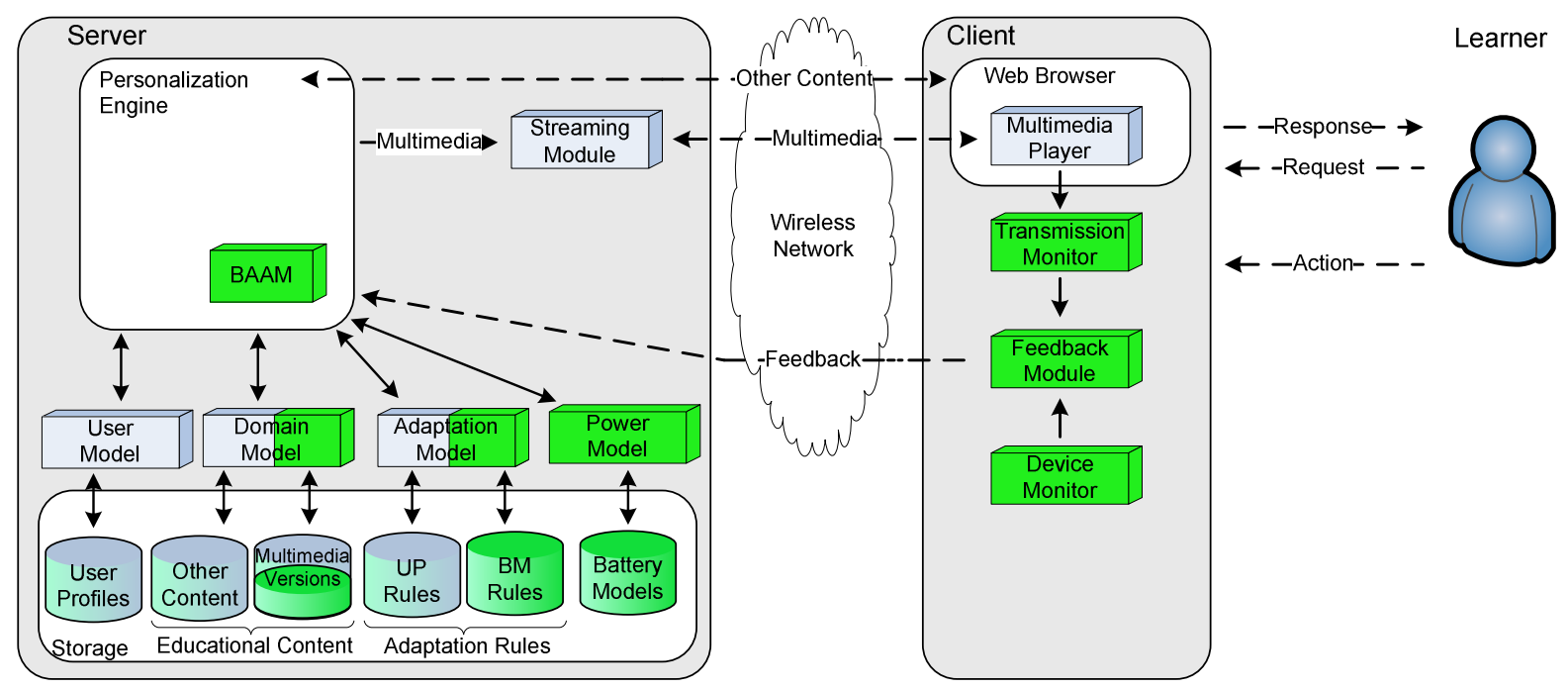

Fig. 3. Battery Aware Adaptive M-Learning System Architecture

device which was used to access the m-learning application at least one time. Multiple BM can be associated to a user, one for each particular mobile device he is using with the mlearning system. A BM contains information such as: device name, battery type, battery model, initial battery capacity, actual battery capacity (the battery was affected by the aging process), battery discharge characteristic, power consumption of different components (e.g. WNIC, CPU, display...) in different situations (e.g. in case of WNIC, power consumption in the four specific states: sleep, idle, receive and transmit), etc. This information is gathered explicit (via registration) or implicit by the Device Monitor (DMon), located on the client side.

PM is updated continuously with new information retrieved through regular feedback from the client, and gathered by Device Monitor (DMon). This information is not saved between user's learning sessions and is not part of BM, but it is used to make an accurate estimation of the remaining battery life in the current consumption conditions.

This information includes among the others: battery level, current drain, battery voltage, battery temperature and is used both to estimate the actual capacity of a battery affected by the aging process and to make a more accurate prediction of the remaining battery life in the current consumption conditions.

Other relevant information for the power saving mechanism, gathered by DMon, is the application load, the device settings, such as screen brightness and sound volume, the connectivity interfaces power state (e.g. Bluetooth), but also user actions that result in the change of these parameters.

The personalisation of educational content is performed by the PE, and consists in the right selection of the atomic concepts from the DM that suits to the learner profile (UM) following the condition-action rules. PE's functionality was extended with a Battery Aware Adaptation Module (BAAM) in order to consider the available power resources of the mobile device in the educational content personalisation process. The personalisation procedure is presented in more details through an exemplification in the next section.
The streaming module is responsible for delivering the multimedia part of the educational content as selected by the PE. It is also controlled by the PE.

On the client side the educational content is displayed using a web browser and / or a multimedia player. The classic architecture of an ALS was extended with a Transmission Monitor (TMon), responsible for retrieving information related to the streaming process and estimating the quality of the video at reception and a Device Monitor (DMon) responsible for retrieving information related to the device status, battery status and user's actions that impact the power consumption. The information collected by DMon and TMon is send periodically to the PE by the Feedback Module (FM). $\mathrm{PE}$ also can receive indirect requests from the learners if they want to see a higher quality for the time the battery will allow them.

\section{B. Example of a personalisation scenario}

This section presents an example of a possible real learning scenario, and how BAAMLS will perform the personalisation of educational content.

When a mobile learner accesses for the first time the learning application, he/she will be requested to register and to provide details about his device and battery model. Using this preliminary information the system will retrieve from various sources, additional details that users are not aware of, such as battery discharge characteristic, to create the device BM.

After registration the learner can proceed further and send a request to access a learning activity. PE will select atomic concepts from the DM that correspond to different pieces of educational content and it will combine them, UP Rules, in such a way to suit to the learner profile (UM).

Battery aware personalisation will be addressed only if multimedia content is selected for a learner. When the learner device starts to retrieve the multimedia stream, the FM also starts to inform the PE with regard to the transmission quality and device power consumption. Based on the information retrieved by the DMon and on the BM for this particular combination user / device model / battery type, PE will 
estimate how much power is still available, but also how much power is needed to play all the multimedia sequences contained by this learning session, with the default properties that offer a good level of quality.

If $\mathrm{PE}$ estimates that there is enough battery power to complete the learning session, no adaptation will be performed. If the remaining battery power is not enough, PE will select a version of the video with reduced properties that still offers a good level of quality but at the same time is more energy efficient, following the BM Rules. If still the battery power is not enough, to complete all the multimedia sequences, but the quality cannot be reduced anymore without affecting the learner's QoE, the version with the highest battery power save from those with an acceptable quality will be delivered.

\section{CONCLUSION AND FUTURE WORK}

This paper presented a new adaptive framework that considers the available power resources of the mobile device, in the personalisation process of the multimedia content delivered to mobile learners. Power Model is one of the main components of BAAMLS, modeling in real-time the mobile device battery and its behavior, and is used to determine the the optimal values for the parameters of a multimedia clip that is being streamed, with the aim of reducing the power consumption while maintaining a good perceived quality.

Experimental tests were conducted in order to determine battery power consumption when a multimedia clip is streamed to a mobile device. Results have shown that the WNIC is responsible for up to $50 \%$ of the total battery consumption when receiving the streamed video. Results have also shown that battery life can be extended by varying some of the encoding parameters such as video compression technique, resolution, frame rate and bit rate. Significant more power can be saved by choosing a more energy efficient compression technique than reducing the quantitative parameters of the video streamed. Preliminary subjective tests have also shown that a controlled variation of the video compression technique has a reduced effect on end user perceived quality, comparing with the variation of quantitative video parameters.

Future work will extend the system's power saving capabilities with new techniques for the other two stages of multimedia streaming process, reception and playing. More elaborate subjective testing will be conducted on a large group of participants in order to determine the effect of each particular power saving technique on the learner perceived quality of the multimedia content. The results will be used to design the personalisation algorithm that maintain the right balance between the quality of multimedia content delivered and the learner perceived quality, in order to extend the battery life and maximize the learning outcome.

\section{REFERENCES}

[1] C. Leung and Y. Chan, "Mobile Learning: A New Paradigm in Electronic Learning", 3rd IEEE Int. Conference on Advanced Learning Technologies (ICALT'03), Athens, Greece, July 9-11, 2003.

[2] A. Bradley, R. Haynes and T. Boyle, "Adult Multimedia Learning with PDAs - The User Experience", The 4th World Conference on mLearning, 2005.

[3] K. Wolf, S. Linckels and C. Meinel, "Teleteaching Anywhere Solution Kit (Tele-TASK) Goes Mobile“, SIGUCCS'07, Orlando, Florida, 2007.

[4] A. Schmidt, "Implicit Human Computer Interaction through Context", Personal and Ubiquitous Computing, vol. 4, 2000, pp. 191-199.

[5] K. Synnes, J. Nord, and P. Parnes, "Location Privacy in the Alipes platform," System Sciences, 2003. Proceedings of the 36th Annual Hawaii International Conference on, 2003, p. 10-19.

[6] F. Meawad and G. Stubbs, "A framework for enabling on-demand personalised mobile learning," International Journal of Mobile Learning and Organisation, vol. 2, 2008, pp. 133-148.

[7] S.A. Petersen and J.K. Markiewicz, "PALLAS: Personalised Language Learning on Mobile Devices," Wireless, Mobile, and Ubiquitous Technology in Education, 2008. WMUTE 2008. Fifth IEEE International Conference on, 2008, pp. 52-59.

[8] C. H. Muntean, "Improving Learner Quality of Experience by Content Adaptation based on Network Conditions", Computers in Human Behavior Journal, Special issue on "Integration of Human Factors in Networked Computing", Vol. 24, Issue 4, pp. 1452-1472, 2008

[9] C. H. Muntean, G.-M. Muntean, "End-User Quality of Experience Aware Personalised ELearning", "Architecture Solutions for E-Learning Systems", C. Pahl (Ed), Idea Group Inc, 2008

[10] G. Anastasi, M. Conti, and W. Lapenna, "Power saving policies for wireless access to TCP/IP networks," Proceedings of the 8-th IFIP Workshop on Performance Modelling and Evaluation of ATM and IP Networks (IFIP ATM\&IP2000), Ilkley (UK), 2000.

[11] J. Adams, G.-M. Muntean, "Power-Dependent Adaptation Algorithm for Mobile Multimedia Networking", IEEE Broadcasting Symposium, Las Vegas, USA, 2006

[12] F. Zhang, S. Chanson, "Proxy-assisted scheduling for energy-efficient multimedia streaming over wireless LAN", 4th Int. IFIP- TC6 Networking Conference, Lecture Notes in Computer Science, Vol. 3462, 2005, pp. 980-991.

[13] Y. Wei, S. M. Bhandarkar, S. Chandra, "A client-side statistical prediction scheme for energy aware multimedia data streaming", IEEE Transactions on Multimedia, Vol. 8, no. 4, 2006, pp. 866-874.

[14] S. Pasricha, S. Mohapatra, M. Luthra, N. Dutt, and N. Venkatasubramanian, "Reducing backlight power consumption for streaming video applications on mobile handheld devices", In Proc. First Workshop Embedded Systems for Real-Time Multimedia, pp. 1117, 2003

[15] H. Shim, N. Chang, and M. Pedram, "A backlight power management framework for battery-operated multimedia systems," IEEE Design and Test of Computers, vol. 21, no. 5, 2004, pp. 388 - 396.

[16] MediaCoder - more than a universal audio/video transcoder. $\mathrm{http}: / /$ mediacoder.sourceforge.net/.

[17] Adobe - Flash Media Server Products. http://www.adobe.com/products/flashmediaserver/.

[18] Darwin Streaming Server. http://dss.macosforge.org/.

[19] VideoLAN - Free and Open Source software and video streaming solutions for every OS! http://www.videolan.org/.

[20] BattLog 0.2.3.130 (Beta) - xda-developers. http://forum.xdadevelopers.com/showthread.php?t=444920.

[21] A.-N. Moldovan and C. H. Muntean, "Increasing Learner's Experience trough Power-Based Adaptation of Educational Content Selection and Delivery," EdTech 2009: 10th Annual Irish Educational Technology Users' Conference, Dublin, Ireland, May 2009. 